\title{
Classifying Workers into Risk Sensibility Profiles: a Neural Network Approach
}

\author{
Beatrice Lazzerini and Francesco Pistolesi \\ Department of Information Engineering \\ University of Pisa, Italy \\ $\{$ b.lazzerini, f.pistolesi\}@iet.unipi.it
}

\begin{abstract}
In this paper we propose a neural network-based classifier to associate a worker with his/her risk sensibility profile. The basic idea behind the risk sensibility profile is that risks are preventable by appropriate actions that decrease their injurious potential. Also, some criticality factors have been shown to be connected with risk perception and risk propensity. Mapping workers into risk sensibility profiles means to measure how safely workers interact with the risks they are exposed to, by considering the preventing actions they perform, and their criticality factors. The main advantages of the proposed classification consist in: (i) supporting the selection of the most suitable worker to safely perform a given task; (ii) tailoring the safety training to each worker's need, to effectively decrease the probability of injury. The proposed neural classifier was trained by using interviews we collected within some volunteer shoe factories. Workers were asked to indicate the preventive actions they would perform if exposed to one or more risks, among a set of proposed actions. Also, workers answered questions to associate a value with each criticality factor. Two typical tasks of the footwear industry, characterized by one and two risks, respectively, were considered to validate and test the classifier.
\end{abstract}

Keywords-classification; neural network; risk; risk perception; risk propensity; risk sensibility.

\section{INTRODUCTION}

Nowadays working environments are still characterized by a huge quantity of risks, and workers are inevitably exposed to such risks while performing their tasks. In the last years, thanks to the ever-increasing interest in safety management, the understanding of the basic concepts related to risk management has been emphasized [1], and, at the same time, several approaches have been proposed to support both risk management and risk assessment [2]-[5]. Accordingly, industrial machineries have been completely redesigned and equipped with increasingly sophisticated safety devices, with the aim of making factories safer places wherein workers are less and less threatened by risks [6], [7]. Notwithstanding, the residual risk remains incredibly high [8]. To safely deal with the risks to which a worker is inevitably exposed to, it is extremely important that he/she understands the risks themselves and, above all, he/she is aware of their hazardous potential.

Two main aspects are related to people's behaviour in hazardous situations: risk perception and risk propensity [9]. Risk perception is the subjective way with which one estimates characteristics and gravity of hazardous situations [10]-[14], while risk propensity is a person's tendency to take or avoid risks [15]-[17]. Many factors influence risk perception, e.g., past experience and knowledge, past health status, psychological, social, political, and cultural factors, mood and emotions, personal knowledge about the risky condition, trust in risk management institutions, age, sex, locus of control [18], [19], optimism bias [19], [20], etc. On the other side, risk propensity is dependent on personality and experience, cultural background, mood, feelings, gender, education, job position, age, etc.

Although the elements influencing risk perception and risk propensity have been widely investigated, behaviours according to which people deal with a given risk actually remain not completely understood. Moreover, correlations between risk perception, risk propensity, and decisions one takes in risky situations are not well known [9]. For the aforementioned reasons, today safety training is adopted to continuously improve workers' ability of promptly recognizing a potential hazard, in order to avoid its negative effects and increase the risk sensibility [21].

Teaching a group of workers the right way of interaction with a given risk is a difficult task, especially if the safety training is carried out in the same way for all the workers, indiscriminately. In fact, by doing so, some workers may not get enough awareness from the training process, keeping inadequate interaction with the risk. A solution to the problem could be to adapt the safety training to the specific worker, through a preliminar classification into a risk sensibility profile.

A risk sensibility profile is an objective way the authors proposed in [22] to measure how safely a worker interacts with one or more injury risks, with the aim of tailoring the safety training to each worker's need, so as to maximize the result in terms of learning. Summarizing our previous work, the concept of risk sensibility profile is based on the fact that a risk can be prevented by performing one or more actions that reduce the probability of the risk to occur. Each action, called preventing action has a level of prevention expressing the goodness of the action in preventing the risk. Risk sensibility profiles are also based on some criticality factors whose correlation with risk perception and risk propensity has been stated by sociology and psychology experts, namely, gender, age, level of education, income, risk knowledge, work control at work site, professional role, injury frequency, effect seriousness, delayed occurrence of 
effects, role repetitiveness, industrial injuries and diseases, acquired skills, perception of risk control, work gratification, state of health, safety culture in the company, anxiety level, self-esteem, worry level [22]. Considered a worker and a risk (or a set of risks) which can be prevented by a set of preventing actions, the risk sensibility profile of the worker with respect to each risk, or set of risks, is characterized by his/her criticality factors and the preventing actions he/she would perform if exposed to that risk, or set of risks.

This paper constitutes the continuation of [22] wherein, in addition to the concept of risk sensibility profile, we proposed an association rule-based technique to mine risk sensibility profiles. More in detail, in this paper we propose a neural network-based classifier to associate a new worker with a risk sensibility profile, selected among the ones previously mined. Through the proposed neural classification it is possible to help a decision maker to choose the most suitable worker to perform a given task which exposes the worker to one or more risks. Also, as previously said, once all the workers are classified in risk sensibility profiles, it is possible to tailor the safety training to each class of workers, by using methods carefully adapted to the level of risk sensibility of the workers belonging to a particular class.

The rest of this paper is organized as follows: in Section II some fundamentals of artificial neural networks and supervised learning are given; in Section III we describe the concept of risk sensibility profile; in Section IV we explain how we perform the classification of a worker into a risk perception profile with respect to a risk, or a set of risks; Section V contains the validation of our approach and the results we obtained for two real tasks; in Section VI we draw the conclusion of our work.

\section{ARtificial NEURAL NETWORKS}

\section{A. Fundamentals}

An artificial neural network (ANN) is a mathematical model trying to emulate the learning-based strategy the human brain uses to solve complex problems [23].

The elementary component of an ANN is the artificial neuron. Hereafter, artificial neurons are simply referred to as neurons. A neuron can be viewed as a node having $n$ inputs and one output. In an ANN, neurons are connected to each other so that the output of a neuron is connected to the input of other neurons. Also, each connection is associated with a weight. There exists a wide variety of architectures of an ANN, i.e., ways to connect neurons to each other.

Undoubtedly, one of the most popular architectures is the multi-layer perceptron (MLP). In MLP neural networks, neurons are organized in layers: one input layer, one or more hidden layers and one output layer. Each layer is composed of a number of neurons that is dependent on the problem. Further, the output $y_{j}$ of the $j$-th hidden or output neuron

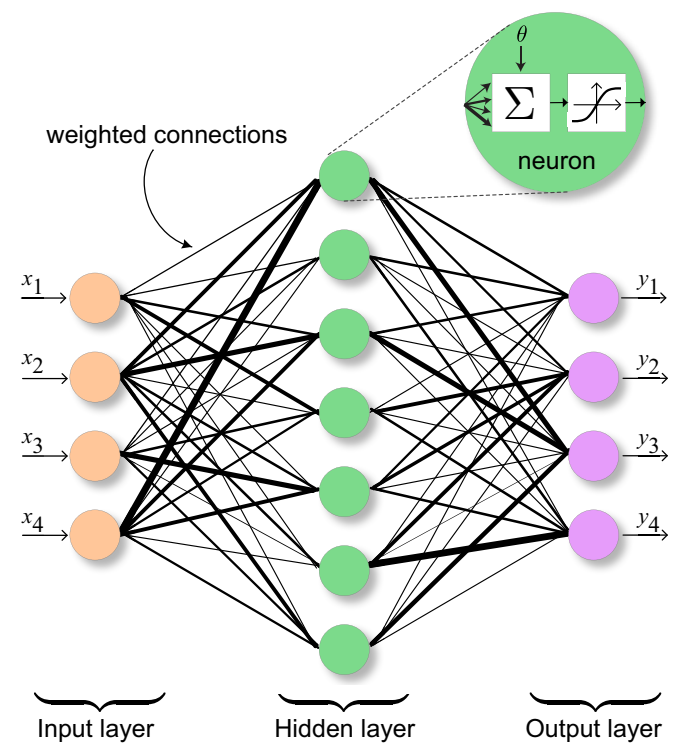

Figure 1. A multi-layer perceptron neural network having four neurons in the input and in the output layers, and seven neurons in the unique hidden layer

is computed as

$$
y_{j}=f\left(\theta_{j}+\sum_{i=1}^{k} \omega_{i j} x_{i}\right),
$$

where $k$ is the number of inputs to the neuron, $\theta_{j}$ is a threshold, $\omega_{i j}$ is the weight associated with the $i$-th input $x_{i}$, and $f$ is a linear/non-linear activation function (typically sigmoid, logistic or hyperbolic tangent). Fig. 1 shows an MLP neural network having four neurons in the input and output layers, and seven neurons in the hidden layer. The weight of each connection is represented by the thickness of the segment denoting the connection itself: the higher the thickness of the segment, the higher the weight of the connection. ANNs wherein information exclusively propagates from the input layer to the output layer, without feedbacks, are called feed-forward. ANNs are able to solve complex problems through the learning process: one of the learning techniques is supervised learning.

\section{B. Supervised learning}

Supervised learning is based on a set of pairs (input, desired_output), called training set. During the learning process, the inputs of the training set are presented to the network, and the error, i.e., the difference between the actual output and the desired output, is measured. By means of a training algorithm (e.g., backpropagation) the weights of the network are progressively adjusted, in order to minimize the global error on the training set. The trained network is tested by comparing the actual output related to input data not contained in the training set with the desired output for such 
data. If the learning process succeeds, the ANN has inferred the unknown relation between the inputs and the outputs, therefore, the trained ANN can be used to effectively predict the output related to new input data.

Some applications of ANNs are approximation of complex functions whose analytical expression is unknown, pattern recognition, classification, and prediction.

\section{RISK SENSIBILITY PROFILES}

\section{A. Overview}

Let us consider a working environment characterized by a set of risks $\mathcal{R}=\left\{r_{1}, \ldots, r_{|\mathcal{R}|}\right\}$, where $|\cdot|$ denotes the cardinality, and a set of preventive actions $\mathcal{A}=\left\{a_{1}, \ldots, a_{|\mathcal{A}|}\right\}$ preventing all the risks in $\mathcal{R}$, that is, any risk $r_{i} \in \mathcal{R}$ can be prevented with a subset of actions $\mathcal{A}_{r_{i}} \subseteq \mathcal{A}$. Also, we consider a set of criticality factors $\mathcal{F}$. Given a worker, each criticality factor is quantified by a criticality score in $[0,1]$. More formally, let $\phi: \mathcal{F} \times \mathcal{W} \rightarrow[0,1]$ be a function associating a value in $[0,1]$ with a criticality factor evaluated for a worker.

\section{B. Single-risk sensibility profile}

The single-risk sensibility profile of a given worker with respect to a given risk is composed by a subset of all the preventive actions the risk can be prevented through, i.e., a specific behaviour toward the risk, and a particular configuration of scores for the criticality factors.

More formally, considered a worker $w$ exposed to the risk $r_{i}$, he/she chooses one or more actions to protect himself/herself from the risk. By denoting with $\mathcal{A}_{r_{i}}^{w} \subseteq \mathcal{A}_{r_{i}}$ the set of preventive actions the worker $w$ would perform to prevent the risk $r_{i}$, the single-risk sensibility profile $\mathcal{P}_{r_{i}}^{w}$ of the worker $w$ for the risk $r_{i}$ is defined as:

$$
\mathcal{P}_{r_{i}}^{w}=\left\{\mathcal{A}_{r_{i}}^{w}, \bigcup_{f \in \mathcal{F}}(f, \phi(f, w))\right\} .
$$

\section{Multi-risk sensibility profile}

In general, a task $t$ exposes a worker to more than one risk. Let $\mathcal{R}_{t}$ be the set of risks the task $t$ exposes to, with $\mathcal{R}_{t} \subseteq \mathcal{R}$. In order to define a multi-risk sensibility profile for a worker with respect to the task, it is important to know which actions are chosen by the worker to prevent each risk of the considered task. To formally represent this concept, we introduce the strategy, i.e., a pair $\left(r_{i}, \mathcal{A}_{r_{i}}^{w}\right)$ meaning that the risk $r_{i} \in \mathcal{R}$ is prevented by the worker $w$ through the actions in $\mathcal{A}_{r_{i}}^{w} \subseteq \mathcal{A}_{r_{i}}$. The multi-risk sensibility profile $\mathcal{P}_{t}^{w}$ of the worker $w$ for the task $t$ is formally defined as:

$$
\mathcal{P}_{t}^{w}=\left\{\bigcup_{r_{i} \in \mathcal{R}_{t}}\left(r_{i}, \mathcal{A}_{r_{i}}^{w}\right), \bigcup_{f \in \mathcal{F}}(f, \phi(f, w))\right\} .
$$

\section{ClasSIFYING WORKERS IN RISK SENSIBILITY PROFILES}

As previously stated, in this paper we use neural networks to classify a worker into a risk sensibility profile both in the case of a single risk, and in the case of multiple risks. In the next two sections we show the architecture of the neural network used in the two cases, describing the inputs and outputs of the network. The number of hidden layers and the number of neurons in each hidden layer will be specified in Section $\mathrm{V}$ as the result of the simulations.

\section{A. Single-risk classifier}

Let us consider a risk $r$ which can be prevented through a set of preventing actions $\mathcal{A}_{r}=\left\{A_{1}, \ldots, A_{m}\right\}$. Also, we take into account a set of criticality factors $\mathcal{F}=\left\{f_{1}, \ldots, f_{k}\right\}$. Note that, in this section, for simplicity, we indicate the single risk we deal with as $r$, thus omitting the $i$ subscripted. For clarity, we recall that, as formally described in Section III-A, each criticality factor $f_{i}$, where $i \in\{1, \ldots, k\}$, assumes a value in $[0,1]$.

Considered a worker $w$ to be classified, he/she is asked to choose which actions in $\mathcal{A}_{r}$ he/she would perform if exposed to the risk $r$. Let these actions be in $\mathcal{A}_{r}^{w} \subseteq \mathcal{A}_{r}$. In order to form the input of the neural network, we consider the vector $\mathbf{a} \in\{0,1\}^{m}$, whose generic element $a_{j}$ is related to the action $A_{j} \in \mathcal{A}_{r}$, and $a_{j}=1$ if the worker to be classified chooses the action $A_{j}$ to prevent the risk, otherwise $a_{j}=0$, where $j \in\{1, \ldots, m\}$.

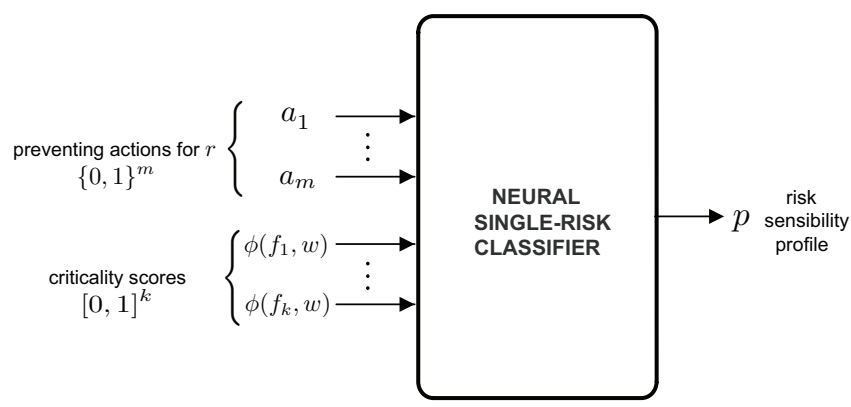

Figure 2. The neural network classifier for the multi-risk classification.

In addition, the worker is asked to answer some questions to assign a score to each criticality factor. In particular, considered a criticality factor $f_{i} \in \mathcal{F}$, the score of the worker $w$ with respect to $f_{i}$ is denoted as $\phi\left(f_{i}, w\right)$, where $i \in\{1, \ldots, k\}$. Finally, let $\mathcal{P}_{r}$ be the set of single-risk sensibility profiles mined, as explained in [22], for a task that exposes to the single risk $r$. Let each profile in $\mathcal{P}_{r}$ be identified by a number $p \in\left\{1, \ldots,\left|\mathcal{P}_{r}\right|\right\}$.

According to the previous considerations, as shown in Fig 2 , the neural network that we propose to classify a worker $w$ into a profile in $\mathcal{P}_{r}$ has $(m+k)$ inputs and one output. Each of the first $m$ inputs is related to a preventing action; the 
other $k$ inputs represent the scores for the criticality factors. The output is simply the profile the worker is classified into.

\section{B. Multi-risk classifier}

If we want to classify a worker with respect to a task that exposes him/her to more than one risks, the neural network has to receive the actions the worker would perform to prevent each risk of the task. In particular, let us consider a task $t$ characterized by a set of risks $\mathcal{R}_{t}$. Each risk $r_{i} \in \mathcal{R}_{t}$ can be prevented by a specific set of preventing actions $\mathcal{A}_{r_{i}}=\left\{A_{1}^{i}, \ldots, A_{\left|\mathcal{A}_{r_{i}}\right|}^{i}\right\}$. Let us consider one vector $\mathbf{a}^{\mathbf{i}} \in\{0,1\}^{\left|\mathcal{A}_{r_{i}}\right|}$ for each $i=1, \ldots,\left|\mathcal{R}_{t}\right|$. Coherently with Section IV-A, the generic element $a_{j}^{i}$ of $\mathbf{a}^{\mathbf{i}}$ is equal to one if the worker to be classified chooses the action $A_{j}^{i}$ to prevent the risk $r_{i}$. The neural network for the multi-risk classification has $\left(\sum_{r_{i} \in \mathcal{R}_{t}}\left|\mathcal{A}_{r_{i}}\right|+k\right)$ inputs composed by the sum of the preventing actions of each risk of the task $t$, and the $k$ criticality factors. As in the single-risk scenario, the network has a single output denoting the profile of the classified worker. The multi-risk classifier is shown in Fig. 3.

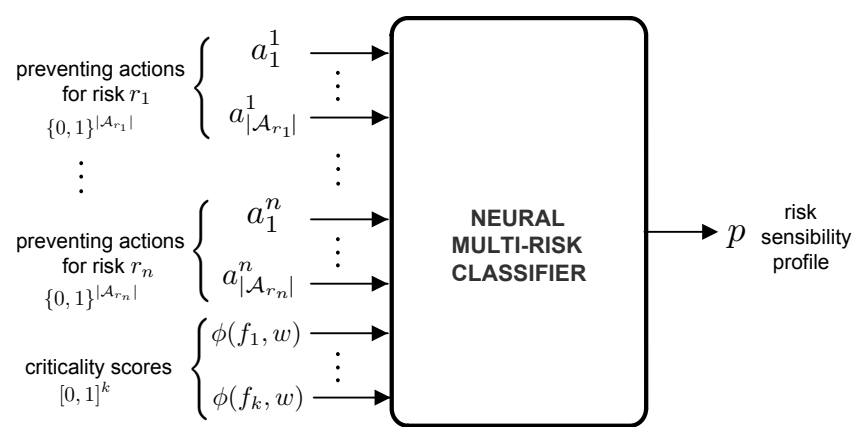

Figure 3. The neural network classifier for the multi-risk classification. In the figure, it is supposed $\left|\mathcal{R}_{t}\right|=n$.

\section{VALIDATION AND DISCUSSION}

\section{A. Dataset}

The experiments we carried out to test the proposed classifier are based on data we collected by interviewing 300 workers of voluntary shoe factories. In particular, the interviews were remotely collected via a questionnaire hosted by a website we purposely created by using the JEE platform. In this way, data collection was performed by ensuring the anonymity of the people that took part in the experiments.

In the questionnaire, we considered two typical tasks of the footwear industry: leather cutting and shoe sole gluing. Leather cutting exposes a worker to a single risk, i.e., the cut risk. On the other hand, shoe sole gluing exposes to intoxication caused by the chemicals emitted by adhesives, and falling. The worker performing the shoe sole gluing task is exposed to the risk of falling whenever he/she has to put the semi-finished product on vertical shelves from which the goods are subsequently shifted by the warehouse workers to the other areas of the factory. For each of these risks we proposed a set of preventing actions, including no action at all. As well as specifying the set of preventive actions to use for each of the considered risks, workers were asked to answer some questions in order to assign a score to a subset of five criticality factors, among the ones introduced in Section I. In particular, we used age, gender, education, past health status, and anxiety level. For measuring the anxiety level we used the Zung anxiety test [24].

The collected dataset consists of 300 samples for the single-risk classifier, and 300 additional samples for the multi-risk classifier. The structure of the samples of each classifier is described in the following. Also, by using the technique proposed in [22] we found five profiles both for the leather cutting task and for the shoe sole gluing task. Profiles are numbered from 1 to 5 , with decreasing carefulness with respect to the risk, or risks. In particular, profile 1 is the safest; profiles 2, 3 and 4 represent behaviours with intermediate level of carefulness; profile 5 is the least safe way of interaction with the considered risk (or risks).

\section{B. Training and architecture of the neural classifier}

Diverse trainings were carried out in the MATLAB ${ }^{\circledR}$ environment to attain the best MLP neural network architecture for the single-risk and the multi-risk classifier. As stated in Section IV, the number of neurons of the input layer is dependent on the classifier, single-risk or multirisk, and it will be discussed, respectively, in Section V-C and in Section V-D. In both cases, the neural network has a single neuron in the output layer, representing the risk sensibility profile of the worker, and a single hidden layer. Each neuron of the hidden layer is based on the hyperbolic tangent sigmoid activation function. Also, a linear activation function was chosen for the output neuron.

To find out the most suitable number of neurons of the hidden layer, we performed various trainings. In particular, the $70 \%, 15 \%$ and $15 \%$ of the dataset described in Section V-A were randomly extracted to form, respectively, the training, test and validation sets. Trainings were performed by varying, with step one, the number of neurons from 5 to 10 , based on heuristic considerations. For each number of neurons, we performed 30 trainings and we computed the average $M S E$ (mean squared error) and the confusion matrix on both the training and the test set, over all the trials. Then, considering all the trained networks with such number of hidden neurons, we chose the one having the best performance, i.e., the minimum MSE on the test set and the most accurate confusion matrix.

\section{Single-risk classification}

As previously stated, for the single-risk classifier we considered the leather cutting task, exposing a worker to the single cut risk. The actions considered to prevent this risk are the following [22]: 



Figure 4. Confusion matrix for the training set and the test set for the single-risk classifier (on the left) and the multi-risk classifier (on the right).

- activation of the machinery safety elements;

- verification of the safety elements efficiency;

- put the gauntlet on;

- keep hands away from the cutting elements;

- switch off the cutting machine to fix a fault;

- periodically check and sharpen the cutting utensils;

- no particular action.

The 300 samples used to train and test the neural network are composed by pairs, whose input consists of 5 binary components, each one related to a preventing action for the cut risk, and 5 components, each one assuming a value in $[0,1]$, for the criticality factors of Section V-A; the output of each sample pair is a number representing the singlerisk profile. In the case of the criticality factor "gender", we represent male with 0 and female with 1 . Also, note that the no particular action choice is represented by setting all the first five components of the input to zero. Summarizing, the neural single-risk classifier we tested for the cut risk has 10 inputs and one output.

By using the simulation procedure described in Section V-A, we attained the best performance with 7 neurons in the hidden layer. The confusion matrix for training and test sets are shown in the left part of Fig. 4. The outputs of the classifier are accurate, as it can be seen by the low numbers in the cells not belonging to to the main diagonal. Further, the MSE on the test set is $8.276 \cdot 10^{-4}$.

\section{Multi-risk classification}

In order to validate the multi-risk classifier, as stated in Section V-A, we considered the shoe sole gluing task. Such task simultaneously exposes a worker to the intoxication risk and the falling risk. To prevent the intoxication risk we considered the following actions [22]:

- activate the extractor fans;

- put the gas mask on;

- avoid to breathe during gas emission;

- no particular action.

The falling risk can be prevented performing one or more of the following actions [22]:

- use of the safety snap hooks;

- check of the platform/ladder stability;

- keep from climbing; 
- keep from moving rapidly;

- no particular action.

In this case, the 300 samples of the dataset have inputs composed by 7 binary components for the preventing actions and 5 components with value in $[0,1]$ for the criticality factors. More precisely, each one of the first three binary components is related to a preventing action of the intoxication risk, and each of the remaining four components is connected to a preventing action of the falling risk. As in the single-risk classification, whenever a worker chooses no particular action to prevent a risk, all the binary components that concern its preventing actions are set to zero. Therefore, the neural multi-risk classifier for the shoe sole gluing has 12 inputs and one output.

Also in this case, the simulation procedure described in Section V-A was used. The highest performing network we attained has 6 neurons in the hidden layer. The confusion matrix for the training and the test sets are shown in the right part of Fig. 4. The classification accuracy, although slightly lower, is comparable to the single-risk classifier and, in this case, the MSE on the test set is $1.138 \cdot 10^{-3}$. However, the errors made by the classifier are related to profiles $(2,3$ and 4) that are very similar in terms of their ability to prevent the risks, since they differ in only one or two preventing actions and they are characterized by almost similar values for the critical factors.

\section{CONCLUSION}

In this paper we proposed a neural network-based classifier to associate a worker with a risk sensibility profile, that is, an objective way to effectively measure his/her carefulness in dealing with one or more risks.

To validate the proposed classifier we used a dataset we collected thanks to 300 voluntary workers. The proposed classifier was validated with respect to two typical tasks of the footwear industry: one of them exposes the worker to a single risk, while the other exposes him/her to two risks, at the same time. Accurate results were obtained both in the single-risk case and in the multi-risk case, with MSE equal to $8.276 \cdot 10^{-4}$ and $1.138 \cdot 10^{-3}$, respectively.

The proposed classification can certainly help to diversify the safety training, by adapting the training method to the particular class to which the workers to be trained are associated, i.e., their risk sensibility profile. A safety training specifically tailored to the worker's risk sensibility can undoubtedly enhance the outcome of the training, if compared to the classic approach wherein the learning method is indiscriminately the same for all the workers. Moreover, the classification of a worker in a risk sensibility profile can support a decision maker to choose the most suitable worker to safely perform a given task.

\section{REFERENCES}

[1] T. Aven, "Foundational issues in risk assessment and risk management," Risk Analysis, 2012, doi: 10.1111/j.1539-6924.2012.01798.x.
[2] D. Dubois, "Representation, propagation, and decision issues in risk analysis under incomplete probabilistic information," Risk Analysis, vol. 30, no. 3, pp. 361-368, 2010.

[3] B. Lazzerini and L. Mkrtchyan, "Risk analysis using extended fuzzy cognitive maps," in Proc. Int. Conf. on Intelligent Computing and Cognitive Informatics, IEEE, Ed., 2010, pp. 179-182, kuala Lumpur, Malaysia.

[4] B. Lazzerini and L. Mkrtchyan, "Analyzing risk impact factors using extended fuzzy cognitive maps," Systems Journal, IEEE, vol. 5, no. 2, pp. 288-297, 2011.

[5] K. B. Misra, "Risk analysis and management: An introduction," in Handbook of Performability Engineering, K. B. Misra, Ed. Springer London, 2008, pp. 667-681.

[6] ISO31000, ISO 31000 Risk management - Principles and guidelines. International Organization for Standardization, 2009.

[7] M. Leitch, "Iso 31000:2009. The new international standard on risk management," Risk Analysis, vol. 30, no. 6, pp. 887-892, 2010.

[8] ISO27001, ISO 27001. International Organization for Standardization, 2005.

[9] M. Keil et al., "An investigation of risk perception and risk propensity on the decision to continue a software development project," The Journal of Systems and Software, vol. 53, pp. 145-157, 2000.

[10] M. Bouyer et al., "Personality correlates of risk perception," Risk Analysis, vol. 21, no. 3, pp. 457-465, 2001.

[11] B. Chauvin et al., "Risk perception and personality facets," Risk Analysis, vol. 27, no. 1, pp. 171-185, 2007.

[12] E. Peters and P. Slovic, "The role of affect and worldviews as orienting dispositions in the perception and acceptance of nuclear power," Journal of applied social psychology, vol. 26, pp. 1427-1428, 1996.

[13] L. Sjöberg, "Factors in risk perception," Risk Analysis, vol. 20, no. 1, 2000.

[14] P. Slovic, "Perception of risk," Science, vol. 236, no. 4799, pp. 280285, 1987.

[15] D. Sharma et al., "A higher-order model of risk propensity," Journal of Business Research, vol. 62, pp. 741-744, 2009.

[16] S. Sitkin and L. Weingart, "Determinants of risky decision-making behavior: a test of the mediating role of risk perception and propensity," Academy of management, vol. 38, no. 6, pp. 1573-1592, 1995.

[17] A. Sueiro et al., "Evaluating risk propensity using an objective instrument," Span J Psychol., vol. 14, no. 1, pp. 392-410, 2011.

[18] M. S. Horswill and F. P. McKenna, "The effect of perceived control on risk taking," Journal of Applied Social Psychology, vol. 29, no. 2, pp. 377-391, 1999.

[19] C. T. F. Klein and M. Helweg-Larsen, "Perceived control and the optimistic bias: a meta-analytic review," Psychology and health, vol. 17, no. 4, pp. 437-446, 2002.

[20] J. Costa-Font et al., "Optimism and the perception of new risks," Journal of risk research, vol. 12, no. 1, pp. 27-41, 2009.

[21] N. Nicholson et al., "Risk propensity and personality," Social Research, vol. 8, pp. 1-33, 2001.

[22] B. Lazzerini and F. Pistolesi, "Profiling risk sensibility through association rules," Expert Systems with Applications, vol. 40, no. 5, pp. 1484-1490, 2013.

[23] S. Haykin, Neural Networks: A Comprehensive Foundation. Upper Saddle River, NJ, USA: Prentice Hall PTR, 1998.

[24] W. Zung, "A rating instrument for anxiety disorders," Psychosomatics, vol. 12 , no. 6 , pp. $371-379,1971$. 